\title{
Construction of Virtual Information Center in Scene Disposition of Unexpected Public Emergency Events
}

\section{Jingxin Zhou, Tao Lu}

School of Management, Dalian University of Technology, Dalian, China.

Email: zhoujingxin@dlut.edu.cn, lutao@dlut.edu.cn

Received December 22 ${ }^{\text {nd }}, 2010$; revised January $16^{\text {th }}, 2011$; accepted January $21^{\text {st }}, 2011$.

\begin{abstract}
Unexpected public emergency events have occurred frequently in recent years. How to deal with these events quickly and efficiently has become an important issue. First, the paper describes the importance of information in scene management of unexpected public emergency events, then points out the weaknesses and shortcomings of current emergency plans. After that a model of virtual information center for unexpected public emergency events is proposed. Finally, the paper describes how to build a virtual information center for assisting emergency work by a case.
\end{abstract}

Keywords: Unexpected Public Emergency Events, Scene Disposition, Virtual Information Center

\section{Introduction}

Recently, humans have suffered many public emergency events, including crashes, explosions, urban fires, etc. These emergency situations occur unpredictably and cause individuals and organizations to shift their focus and attention immediately to deal with the situation based on exact information. Therefore, the effectiveness of information delivering plays an important role in the handling of the emergency events.

Various departments perform their own responsibility in emergency incident according to their respective rules and regulations, which can not ensure the fine communication and coordination among various departments. Therefore, the departments need to ensure information delivering straightway in time through unblocked communication with other departments while doing their own work. According to the past experience of handling of emergency incidents, effective communication can reduce the disaster losses to a minimum. However, there are often two problems when communicating with others under tense situation, one is information overload problem [1], namely the information receiver receive far beyond its processing capacity of information, another is information hunger problem, namely people who processing disaster information ability tend not to deal with the information, or people who possess disaster informa- tion don't have ability to handle, even causes information derogations because of bureaucracy system [2]. Therefore, in order to finely solve public emergencies, we must ensure the flow of information is smooth, and guarantee agencies can promptly receive the required information completely, enhance effective communication and coordination thereby.

Scene disposition, as a crucial part in emergency events disposition, refers to carry out site vigilance and rescue work, evacuate masses, control the site, maintain orders and manage other relevant work in the scene after the emergency incident occurs [3]. The scene disposition needs to be deal with immediately and there is no enough time to hold a meeting to arrange. This type of the information particularly emphasis on current situation and can't be arranged uniformly [4]. For the information transferring, we should prepare and drill beforehand and see how the wind blows when we do it.

The current emergency plan of the public emergency is a management plan or an overall plan setting up through a certain procedures before the incidents occur $[5,6]$. It clearly define who is responsible for what to do, when to do, how to do, and the corresponding strategy and resource allocation, etc during public emergencies' preparing and processing $[7,8]$. However, various situations in scene disposition are unpredictable and the information in scene disposition is real-time and trivial, so 
the key problem is the way how information transfer in the process can't be accounted in emergency plans. Therefore, the purpose of this paper is to construct an information center aimed at information collecting and transferring in public emergency events disposition, especially in the scene disposition.

The information involved in the incident is physically dispersed [9]. When the emergency occurs the information is combined to be a system which has a uniform dispatch. It seems like there have an organizational entity called information center, but the information center raised in our paper is a virtual organization without actual existence of office space, so the information center is named as virtual information center compared to the traditional information center. Once Tung X. Bui raised the virtual information center to operate for an emergency disposition in 2001 [10]. This paper focuses on the functions, components and workflow modeling method of virtual information center in scene disposition on the base of reference.

In summary, the virtual information center can handle the transmission of information in emergencies disposition, solve coordination radically in scene disposition of emergency among departments, and thereby reduce the harm extent of accident. This paper mainly researches on how to model the virtual information center and how it works.

\section{Conception of Virtual Information Center}

The concept of virtual information center is first proposed by Tung $\mathrm{X}$. Bui, who considered the virtual information center can effectively deal with large quantities of information request in disaster preparation, disaster management and disaster recovery. The center is independent in certain areas, and is easily connected with government and proposed other organizations which need supportive decisions through the technology of network.

In this paper, we introduce the conception of virtual information center to the field of unexpected emergency event disposition. However, compared to the original definition of virtual information center raised by Bui, the virtual information center in our paper focuses on the scene disposition and we should pay more attention on information delivering, handling and feedback by institutions or people. Decision makers, scene staff and other related personnel can communicate through this information institution timely so as to improve the management efficiency, and drop the disaster loss to the minimum, involve accidents information, influence and the progress of relief work, casualties, etc.

In this paper, the virtual information center is defined as a system that communicates information and consists of ECA rules [11], expert knowledge base and relevant information warehouse and the function of these parts will be illustrated in section3.1. The responsibility of the center is to collect data, information of all areas and data warehouse relevant with crisis utilizing subsistent network communication technology. When unexpected public emergency event occurs, the center provides information support for events disposition according to a certain pattern, result in resolving disaster problem effectively.

Compared with traditional business process, the workflow under emergency has large amounts of dispersive sources, which exchange mass complicated data and information such as device information, product information, operator information, etc. Due to this, the virtual information center should have the following four features:

1) Distribution

Sources of information in virtual information center are distributed dispersedly and they perform their daily work conventionally.

2) Concentration

Virtual information center, as a center, is mainly due to its function as an information platform to store information which is physically dispersed. The information center fully saves time in information gathering in public emergency cases because of information concentration, thus ensure the quick emergency response.

3) Virtuality

Virtual information center is a virtual organization, it doesn't have actual existence of office space, and its office space is established along with the sudden public events.

4) Real-time

Virtual information center receives event information dynamically, and then react to it, so its work flow is not fixed. It also continues receiving new real-time event information and making new workflow along with the emergency disposition.

Constructing a virtual information center demands the mobile devices of perfect function, a network of command and communications, and the best of managerial skills. The explosion of telecommunications technology, the ever expanding Internet, the availability of inter-platform connectivity software made in group decision and negotiation are all making the concept of the virtual information center for emergency disaster relief to be an effective and feasible measure [12].

This chapter describes the definition, characters and necessity of virtual information center in unexpected emergency events. Then we will illustrate how the virtual information center ought to be and how it works.

\section{Construction of VIC in Unexpected Emergency Event}

In the process of constructing virtual information center, 
we should consult existing emergency plans to design work flows and develop corresponding information support systems so as to construct a virtual information center based on it. Through the platform, the system can deliver corresponding commands and inform the target people by collecting information fed back from the sensors or people so as to promise the effective information communication in the public emergency. Virtual information center involves all the departments related in the scope of application from obtaining the warning information till to the incident having been solved, even to the post-processing, but the primary step is the stage of scene disposition.

The virtual information center should organize drills after its construction, finding problems and modifying it in time, to ensure its feasibility and effectiveness. Once the emergency takes place, the center can make effective disposal immediately so as to reduce the possible loss to the least. Constructing the virtual information center overall involves two steps and they are introduced in Sections 3.1 and 3.2, respectively.

The most important function of this virtual information center is collecting and delivering information. The types of the collected information include the information daily collected and the information collected in the scene. Apart from this, a big challenge in delivering information is to send the right information to the right person by proper manner at the right time. In order to solve this problem, the virtual information center needs a suitable workflow mechanism, which is defined by rules and rules can be defined by experience or events. In addition, the most important character of the mechanism for this virtual information center is that the information is delivered by the knowledge, which is stored in the form of ECA rules. Through the rules the system can send corresponding orders or information on the basis of different conditions when various events triggering in emergency. The specific presentation and necessity of introducing ECA rules is illustrated in section 3.3.

\subsection{Basic Components of VIC}

Virtual information center consists of three parts. They are virtual information management system (VIMS), expert knowledge base (EKB), event triggered rule base.

Virtual information management system (VIMS) is mainly responsible for managing and invoking in the system's database, and it stores the information captured by sensors to the database of the information system. The VIMS can also invoke and share data of expert knowledge base, and hand down strategic decisions made by expert knowledge to each department, aimed at directing the relief work in emergency. Therefore, the VIMS is the core of the whole system.

Expert knowledge base (EKB) is mainly responsible for the system's decision analyzing. Censors collect large amount of information after emergency occurs, and EKB filter the information needed by decision analyzing from vast information to prevent information overload, after that, the EKB gives optimal scheme of processing and rescuing decision support through analyzing of public emergencies. The emergency plan compiled beforehand in traditional working process can be seen as a kind of decision knowledge stored in EKB, in addition to some other forms of artificial intelligent algorithm and rules recommend by experts can be stored as knowledge in EKB. The EKB in the whole system plays a high-level decision supported role.

Event triggered rule base is mainly responsible for firing and invoking other modules (such as VIMS, EKB) in virtual information center through beforehand defined event triggering rules. Because in the conventional workflow, the event state is consecutive and connected in the form as flow, while in the unconventional workflow, many unexpected event happens which makes the event state becomes inconsecutive and many independent branches exist in the workflow. Thus, after an emergency occurs, it could initiatively start rule and control the incident conducting timely. These events triggered rules of system are stored in active rule engine (ARE) as the form of ECA rules [13], the ECA rule is traditionally performed as a kind of rule used for the description of discrete event model. ECA rules will be illustrated in next part. The whole structure of the virtual information centre is illustrated in Figure 1.

The focus of this architecture is on how to perform the information matchmaking mechanism in emergency incidents, that is, how to arrange the proper information to the appropriate one at right time.

\subsection{Workflow Modeling}

The process of modeling workflow defines all the process, from the preparing work to finishing work, including process-related information and the status of each instance of the process as it gets executed. The workflow of virtual information center can be divided into two types: conditional workflow and unconditional workflow. We will illustrate each step as follows:

\subsubsection{Preparing Work}

Before the virtual information center starts after the occurrence of emergent events, we need some preparing work. In the normal state, the dairy work of virtual in formation center includes:

1) The construction and maintenance of the sensors, mobile equipments and wireless communication network, etc. Flexible and powerful mobile equipments are guaranteed for the effective handling emergency due to the staff participating in handling emergency are scattering in geography, so the hardware we construct must support coordinated environment based on information, making 


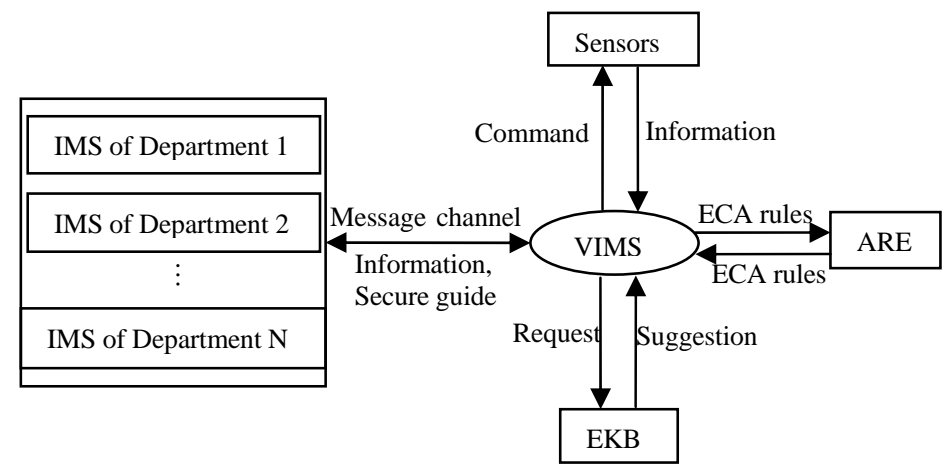

Figure 1. The whole structure of virtual information center.

sure that the information in solving the emergency can be unblocked timely. The hardware must support the six tasks including detecting and reporting task, identifying task, informing task, organizing task, executing task, evaluating and researching task [14].

2) Collecting, sorting and saving information related in solving the public emergency, ensuring the information can be updated in systems timely. The information including the information received by sensors in the emergency scene, information concerning with management departments and information used for decisions. The information storeroom in virtual information center can be divided into five effective information fields which are communicating information, map information, environment information, schedules and procedure information [15].

Resource information consists of the information about the category, quantity and allocating method of emergent traffic equipments, machines and facilities. The workers can get needed resource or find out related connection information immediately through the information, then accelerate the emergency disposition.

Communicating information is the information through which the system contacts with the needed workers to solve the problems when the emergency takes place, which includes telephone numbers, Emails, job responsibilities and available ability that day. The available ability information is influenced by location, weather, health condition, etc.

Map information is the information that ensures to draft the optimal route under emergent situations. We need different route information in different emergent situations such as earthquake, flood, bridge collapse, etc. The kind of information includes location information, optional traffic route information, traffic information and even disposing information of heavy mechanical machines, etc.

Environment information includes local wind direction, wind velocity, temperature, pressure, humidity, rainfall and water, soil information in polluted area. The infor- mation will have positive or negative influence on resource collocating, staff contacting, route and traffic, fire spreading and toxic gases scattering, so we choose the optional information according to the environment information to some degree.

Schedule and procedure information is the information that is formulated ahead of time to ensure handling the emergency safely, and we should draw up the kind of information into the virtual information center while we draft different emergency plans. The information consists of procedure for alerting dangers, specific emergent action plan, system commanding plan and comprehensive emergency management plan. According to the type of information, the staff in the scene can work under the accurate information guiding so as to decrease potential errors.

With the improvement of the emergency job's requirement, we must ensure the accuracy of the ability to obtain, analyze and handle information in the virtual information center, clear and definite procedures to check and ratify information and assure the unity of the information collected, avoiding the contradictory information.

\subsubsection{Unconventional Workflow}

According to the above step, we can deal with the normal workflow through the hardware and the information collected, however, the workflow of the emergency changes dynamically most times. Therefore, the next step we should design the workflow in the non-normal state, that is, after emergency takes place. The entire workflow of the organization is after receiving warning information, the system firstly analyses and verifies information; and then makes decisions according to the guidance from ex-incorporating ECA rules; furthermore, the system sends the commands information to the related departments to instruct their job; finally, the system receives the information feedback from the scene continuously along with the handling with incidents, and then evaluates again, and lastly make the next commands, thereby, control the changes of incidents in time. The specific 
unconventional workflow of the virtual information centre is shown in Figure 2.

We can see that the work of virtual information center is to let the departments gain the accurate and timely information through strengthening the management of sharing information. The system collects and distributes the targeted information to the targeted groups, which not only reaches to "soft" control effect but also has a function of reassuring the people. In this case, what to distribute, how to distribute and when to distribute is becoming complex and detailed decision problems, and this is what the virtual information center should do. The aim to constructing the virtual information center is to fasten the information broadcast and improve the accuracy and velocity of handling the emergency, so the flow of information should be unconstrained, real-time and correct.

\subsection{Workflow Modeling Based on ECA Rules}

After constructing the system architecture and workflow of virtual information center, we need to define the corresponding triggering mechanism considering the urgency and dynamics of public emergencies. This triggering mechanism is based on information interactive level, therefore, we can define the triggering mechanism of public emergencies' virtual information center for corresponding information. This paper applies the triggering mechanism based on ECA rules. It invokes each abstract information module using the initiative and timeliness of ECA rules, and then achieves the effective management of the corresponding personnel or organizations of each module, through that it can control working process in virtual information center.

Event-Condition-Action Rule abbreviates to ECA rule, and initially been used in database sharing field on the purpose of providing the fast reaction of event-driven ability for active database system [16,17]. Many application fields consider and adopt it as one of the most efficient coordination approach because of the concise, clear characteristics in describing the response behavior.

In next section, we will use a case to explain how to use the ECA rules and what the form of the rules.

\section{Case Study}

There are many dangerous chemicals in the chemical laboratory, and leakage, fire, explosions and other causes may result in great risk of chemical accidents which are harmful to society, including acute poisoning, injury or death. Take the chemical accident in chemical lab of one school as an example, this paper will introduce how to build a virtual information center of this accident according to the proposed method of Virtual Information Center in Section 3. In order to build this organization, we need to construct its system architecture based on emergency plan [18] of chemical accident, define the roles and design its workflow, establish the corresponding information support system. The specific scheme is as follows:

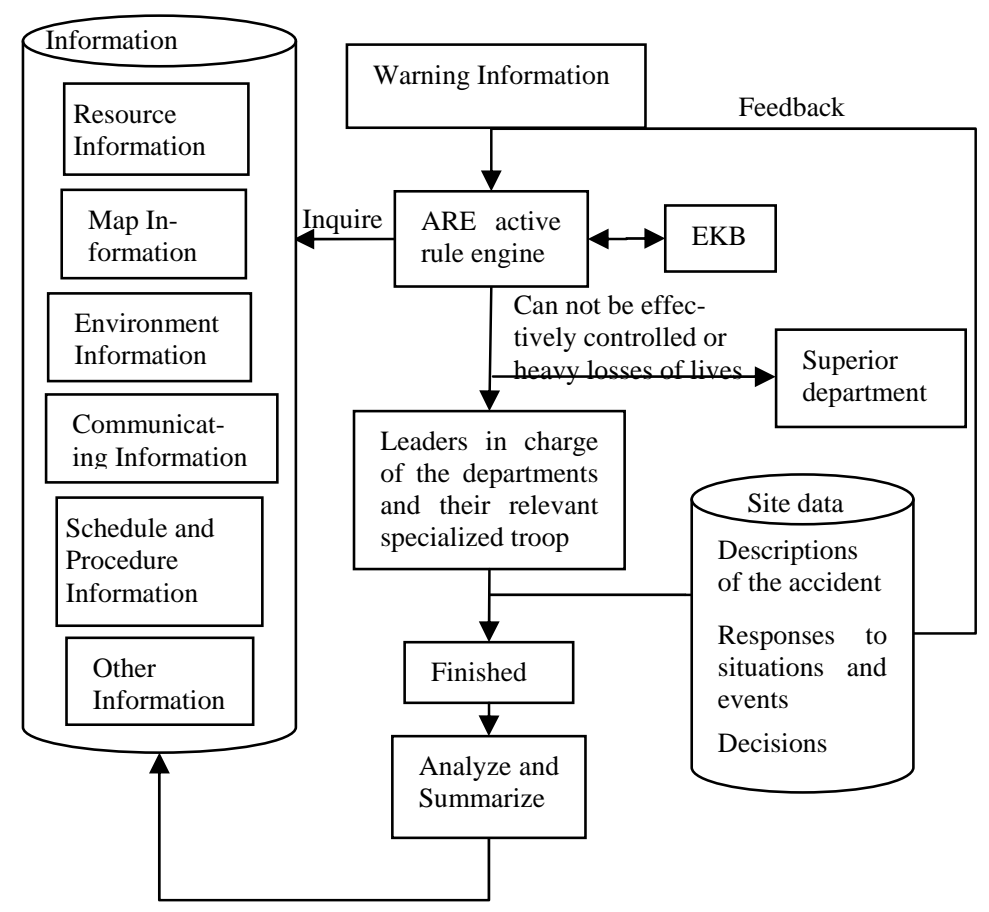

Figure 2. Unconventional workflow of virtual information center. 


\subsection{System Structure Design}

VIMS’ responsibilities are as follows: receiving warning information, instant information from the scene and instruction information of emergency rescue from local government or higher education authorities; submitting information to the decision-makers timely; querying information from the information base and sending messages and commands to each functional department on the purpose of directing and coordinating their rescue work; sending up the situation of the accident and rescuing process to higher education authorities on the condition that the incident can't find effective control or result in heavy casualties.

EKB: The president or the person appointed by president holds the post of chief commander while the vice-president or heads of the departments hold the post of vice-chief in decision-making. The system directs emergency disposing work real-time after receiving emergency information and in the end organizes the accident investigation to update information base. Expert teams needed by EKB are made up of the experts in police stations, fire brigades, health bureaus, environmental protection agencies, transportation bureaus, meteorological bureaus and bureaus of civil affairs. They propound emergency plan and safety measures for emergency rescue, and provide technical guidance for rescue work. All these emergency disposal plans and experts' advices are stored in EKB in the form of rules or knowledge.

IMS of departments:

Public Security Office of School and Handan Municipal Bureau: they formulate pre-arranged planning of personnel evacuation and accident alert. Organization accident may endanger the personnel evacuation in area, so they must make security administration for the personnel evacuation area; hey control the ambient traffic which surrounding the scene of the accident, and they inhibit cars from going into the dangerous area in order to protect the smooth flow of relief road; they involve in the accident investigation and handling.

Hospital Wing and Handan Municipal Public Health Bureau: they formulate pre-arranged planning of treatment of injured person and ambulance emergency. They determine injured person's professional treatment and ambulance designated hospitals, they train corresponding health-care professionals; they direct designated hospital to lay up corresponding medical instrumentation and first-aid medicine; they deploy medical personnel, medical instrumentation and first-aid medicine of the scene, organize rescue and transfer the wounded; they count the case of casualties.

Police Station and Fire Brigade: they formulate pre- arranged planning of handling hazardous chemical accidents. They are in charge of some tasks at the emergency scene, for example detect, vigilance, rescue, risk control, fire extinction, flood protection, export and transfer, decontamination and cleaning.

Environmental Protection Bureau: they organize the monitoring of dangerous chemical pollution incidents, and determine the composition of hazardous substances and the concentration of influential area; they warn the area that may have environmental impact for a long time, put forward controlling measures and monitoring; after the accident under control, they direct hazardous substances remained at the scene to remove the environmental pollution; they survey major pollution incidents of dangerous chemicals and ecological damage events.

Handan Municipal Communications Bureau: they specify emergency transport unit; they supervise maintenance of emergency vehicles, training of drivers, transport goods and materials and workers at the emergency scene.

Handan Municipal Meteorological Bureau: they report weather information and provide corresponding technical support; they provide weather information for emergency scene, such as wind direction, wind speed, temperature, air pressure, humidity and rainfall amount; they organize professional teams to predict the spread of fire, and the direction, speed, scope of toxic gas diffusion.

Handan Municipal Civil Affairs Bureau: they organize the deployment of rescue equipment and goods and materials, transfer and place disaster victims, and implementation of disaster relief life.

Information Office: they organize and coordinate publication of accident news.

School Affairs Office: they deal with the disposal of auxiliary field, such as placating the victims and students timely, contacting with the family of wounded.

\subsection{Workflow Design}

In the view of the dangerous accident in school chemistry lab, the workflow of constructing virtual information center in general is as follows:

1) Constructing and maintaining the sensors, mobile devices, wireless communication network and other hardware facilities. Dangerous chemical accidents mostly have two categories including leaking accidents and fire (explosion), so sensors include automatic fire alarm and automatic alarm sensor which is distributed within the laboratory or around the laboratory to measure the humidity, temperature and concentration of toxic gas in air. Mobile devices include mobile telephones, interphones and other equipments which can receive information. Wireless communication network makes up of computers, a large number of microelectronic devices, precision 
machinery and equipment, etc.

2) Collecting, aggregating and storing the related information mentioned in information base of the previous models, and ensuring to update this information timely in the system. Specific information including:

Resource information includes the information of medical staffs in designated hospitals, medical equipments and first-aid medicines, emergency vehicles of designated emergency transport units, rescue equipments and other materials information, such as categories, amounts and ways of supplying.

Staff communication information includes telephone numbers, emails, positions and available information of members in the expert teams, principals of various functional departments and their professional team members; it also includes the contact information and other message files of teachers, students and their parents (such as family medical histories, date of birth and so on).

Cartographic information is the school's location information, optional routes of police cars, fire trucks, ambulances, transporting vehicles, traffic information, the deployment information of heavy machineries and equipments.

Environmental information is the weather information in the school location of wind direction, wind speed, temperature, atmospheric pressure, humidity, rainfall amount and the pollutant information of water bodies and soil around laboratories.

Plans and procedures information concentrates all program information in alarm reporting procedures, evacuations and alerting plans, treatment and rescuing of the injured persons plans, handling hazardous chemical accidents plans and disposal of hazardous chemical pollution incident plans.

When criminal cases or catastrophic incidents of chemicals happen, the compiling workflow of school's virtual information center is: firstly, receiving warning information by the sensor at the scene or the phone from teachers and students at the scene, including time, place, type, strength and damage of the accident; secondly, launching emergency disposition according to sphere, level and variation of this event immediately after confirming and analyzing warning information; at last, transmitting warning information to the principal of campus office, public security office of school, police station, fire brigade, hospital, health bureau, environmental protection agency, transportation bureau, meteorological bureau and civil affairs bureau, and the heads of these departments organize professional teams and related members to the scene to work. With the processing of the incident, the system receives the information of progressing, accident investigating information, casualties' information, substance transporting information, evacuation information and environmental information fed back from emergency scene by the principals of functional departments or the members of the professional teams. The system makes command of next step after re-evaluating the event.

After disposing the event, organizing personnel to analyze this event and composing information report to modify the pre-compiled information database in virtual information center so that they can dispose emergency work easily in future. According to the format of ECA rules, the instance described above can be represented as the set of rules which are partly shown in Figure 3. Each rule, when the condition is satisfied, will execute an action.

In a word, the virtual information center proposed in this paper can ensure the decision-makers and expert groups to receive feedback information from scene immediately as soon as the chemistry lab accidents happen. We can also send the order and message to the relative target people through virtual information center so that they can take appropriate action in quick response. In this way, we can guarantee the efficiency of scene disposition in chemical laboratory accidents.

\section{Conclusions}

In emergency management, the information is a bridge to link each department and the steps within each department. To make sure that dealing with emergency is effective during a given period of time, we must ensure the

\begin{tabular}{|l|}
\hline On extEvent(sensor1.tempeture > 80) and extEvent(sensor 2.alarm = on) \\
If it is not practice \\
Do creatExtEvent(fire(conten='chemical lab is on fire,hurry!')) \\
\hline On extEvent(fire) \\
If fire.reason = 'Mg burning' \\
Do invokeService(EKB.request(reason of 'Mg burning')) \\
\hline On svcEvent(EKB.suggestion()) \\
If message channel.open() \\
Do creatextEvent(Dep1.prepare (medicine)) and creatextEvent(Dep2.prepare(secure_equipment)) \\
\hline
\end{tabular}

Figure 3. The ECA rules of the part process in this case. 
timely information unblocked in scene disposition.

The current emergency plan, which is adopted in our architecture, is nowadays regarded as the most pervasive method for emergency application integration, however, it assumes huge message exchange over the disposition, and it has evolved with a variety of sensors in wide areas. Furthermore, the ubiquitous paradigm requires more complicated and reactive communication architecture beyond the existing architecture. Due to this, our paper presents a virtual information center referencing on the basis of former. Our approach was proposed to assist the information transferring for unexpected public emergency events. We described the operation mechanism based on ECA rules by improving and deeply discussing the concrete implementation tache. As a result, the primitive people or events, such as sensors, can also interact with information systems to enact collaborative and effective business process.

\section{Acknowledgement}

This research work was supported by the National Natural Science Foundation of China (Grant No. 70771017).

\section{REFERENCES}

[1] A. M. Schaafstal, J. H. Johnston and R. L. Oser, "Training Teams for Emergency Management," Computers in Human Behavior, Vol. 17, 2001, pp. 615-626. doi:10.1016/S0747-5632(01)00026-7

[2] J. Hale, "A Layered Communication Architecture for the Support of Crisis Response,” Journal of Management Information Systems, Vol. 14, No. 1, 1997, pp. 235-255.

[3] Meizhou government, "Scene Disposition Standard of Emergency Event in Meizhou City,” 2009. http://www.mei zhou.gov.cn/zwgk/fggw/sfbgswj/2009-09-30/1254281818 d507 27. html.

[4] N. Wang, S. Han, H. Li and Y. Z. Wang, "Research on Information Integrating Method Based on Entity-Relation-Problem Modeling System," Journal of Dalian University of Technology, Vol. 47, No. 2, 2007, pp. 295-297.

[5] L. Ji, H. Chi and A. Chen, "Emergency Management," Higher Education Press, 2006.

[6] J. J. Xing, "Emergency Plan Compiling Technology in Serious Accidents,” Journal of Chinese Security Science, Vol. 14, No. 1, 2004, pp. 57-60.

[7] D. Z. Peng and J. Y. Hu, "Emergency Plan Research in City Major Accident,” Industrial Safety and Environmental Protection, Vol. 30, No. 2, 2004, pp. 38-40.

[8] H. Jiang, "Scenarios of Evolution in Rare Major Emer- gency Real-Time Decision-Making," Journal of Huazhong University of Science and Technology (Social Science Edition), 2009.

[9] H. Artman, "Team Situation Assessment and Information Distribution,” Ergonomics, Vol. 43, No. 8, 2000, pp. 1111-1128.

doi:10.1080/00140130050084905

[10] T. X. Bui and S. R. Sankaran, "Design Considerations for A Virtual Information Center for Humanitarian Assistance/Disaster Relief Using Workflow Modeling,” Decision Support Systems, Vol. 31, No. 2, 2001, pp. 165-179. doi:10.1016/S0167-9236(00)00129-9

[11] J. Y. Jung, J. Park, S. K. Han and K. Lee, “An ECA-based Framework for Decentralized Coordination of Ubiquitous Web Services," Information and Software Technology, Vol. 49, 2007, pp. 1141-1161.

doi:10.1016/j.infsof.2006.11.008

[12] H. Y. Mak, A. P. Mallard, T. Bui and G. Au, "Building Online Crisis Management Support Using Workflow Systems,” Decision Support Systems, Vol. 25, 1999, pp. 209-224.

doi:10.1016/S0167-9236(99)00007-X

[13] J. Kong, J. Y. Jung and J. Park, “Event-Driven Service Coordination for Business Process Integration in Ubiquitous Enterprises,” Computers \& Industrial Engineering, Vol. 57, 2009, pp. 14-26.

doi:10.1016/j.cie.2008.08.019

[14] Y. F. Yuan and B. Detlor, "Intelligent Mobile Crisis Response Systems," Communications of the ACM, Vol. 48, No. 2, 2005, pp. 95-98.

doi:10.1145/1042091.1042097

[15] S. W. Yoon, J. D. Velasquez, B. K. Partridge and S. Y. Nof, "Transportation Security Decision Support System for Emergency Response: A Training Prototype,” Decision Support Systems, Vol. 46, No. 1, 2008, pp. 139-148.

_doi:10.1016/j.dss.2008.06.002

[16] N. Bassiliades and I. Vlahavas, "Device: Compiling Production Rules Into Event-Driven Rules Using Complex Events," Information and Software Technology, Vol. 39, No. 5, 1997, pp. 331-342.

doi:10.1016/S0950-5849(96)01155-X

[17] U. Dayal, B. Blaustein, A.P. Buchmann, S. Chakravarthy, D. Goldhirsch, M. Hsu, R. Ladin, D. McCarthy and A. Rosenthal, "The HiPAC Project: Combining Active Databases and Timing Constraints," Acm Sigmod Record, Vol. 17, No. 1, 1998, pp. 51-70.

[18] Kunming government, "Dangerous Chemicals Emergency Plan of Kunming,” 2007. http://www.ketdz.gov.cn/ Regulations/ShowArticle.asp?ArticleID =1971. 